\title{
Anxiety risk SNPs on chromosome 2 modulate arousal in children in a fear generalization paradigm
}

\author{
Julia Reinhard ${ }^{1}$ (1) $\cdot$ Carsten Drepper $^{1} \cdot$ Heike Weber $^{2,3} \cdot$ Miriam A. Schiele $^{4} \cdot$ Katharina Kneer $^{1} \cdot$ Anna Mittermeier $^{1}$. \\ Lillien Frey ${ }^{1} \cdot$ Andreas Reif $^{3} \cdot$ Paul Pauli $^{5} \cdot$ Katharina Domschke ${ }^{4} \cdot$ Jürgen Deckert ${ }^{2} \cdot$ Marcel Romanos $^{1}$
}

Received: 22 September 2019 / Accepted: 11 December 2019 / Published online: 21 December 2019

(c) The Author(s) 2019

\begin{abstract}
Alterations in fear learning/generalization are considered to be relevant mechanisms engendering the development of anxiety disorders being the most prevalent mental disorders. Although anxiety disorders almost exclusively have their first onset in childhood and adolescence, etiological research focuses on adult individuals. In this study, we evaluated findings of a recent meta-analysis of genome-wide association studies in adult anxiety disorders with significant associations of four single nucleotide polymorphisms (SNPs) in a large cohort of 347 healthy children (8-12 years) characterized for dimensional anxiety. We investigated the modulation of anxiety parameters by these SNPs in a discriminative fear conditioning and generalization paradigm in the to-date largest sample of children. Results extended findings of the meta-analysis showing a genomic locus on 2 p21 to modulate anxious personality traits and arousal ratings. These SNPs might, thus, serve as susceptibility markers for a shared risk across pathological anxiety, presumably mediated by alterations in arousal.
\end{abstract}

Keywords Anxiety disorders $\cdot$ Childhood and adolescence $\cdot$ Anxious personality traits $\cdot$ Fear conditioning and generalization · Arousal $\cdot$ Anxiety risk genes

\section{Introduction}

Electronic supplementary material The online version of this article (https://doi.org/10.1007/s00787-019-01458-7) contains supplementary material, which is available to authorized users.

Julia Reinhard

Reinhard_J@ukw.de

1 Department of Child and Adolescent Psychiatry, Psychosomatics and Psychotherapy, Center of Mental Health, University Hospital of Würzburg, Würzburg, Germany

2 Department of Psychiatry, Psychosomatics and Psychotherapy, Center of Mental Health, University Hospital of Würzburg, Würzburg, Germany

3 Department of Psychiatry, Psychosomatic Medicine and Psychotherapy, University Hospital of Frankfurt, Frankfurt, Germany

4 Department of Psychiatry and Psychotherapy, Medical Center, Faculty of Medicine, University of Freiburg, University of Freiburg, Freiburg, Germany

5 Department of Psychology (Biological Psychology, Clinical Psychology and Psychotherapy), University of Würzburg, Würzburg, Germany
Anxiety disorders (AD) represent the most prevalent mental disorders [1] and are typically characterized by an early onset in childhood [2]. AD often persist from childhood into adulthood [3] and are the precursor to a range of other psychiatric disorders, e.g., depression [4]. Since pathological anxiety has long-term negative consequences for child development [5], advancing our understanding of the pathogenic, as well as risk factors and mechanisms of AD, has substantial societal impact by defining concrete points of intervention, and thus ultimately improving treatment efficiency, as well as in aiding preventative and treatment efforts.

In the development of manifest $\mathrm{AD}$ many factors play important roles, among others especially personality, environmental, and genetic factors. Although there are different diagnostic definitions relative to their clinical presentation, $\mathrm{AD}$ as a group is believed to have a common underlying diathesis relating to an abnormal threat-response regulation [6]. Unlike categorical definitions, which could be afflicted, e.g., with threshold problems, AD can also be considered on a dimensional continuum with respect to symptom intensity, 
severity, and frequency of occurrence of symptoms and assessed self-report questionnaires symptoms [7, 8].

The National Institute of Mental Health Research Domain Criteria (RDoC) [9] exemplified a change in the way of classifying psychopathology by cutting across traditional nosological divisions, rather than cluster signs and symptoms. Dimensional constructs for studying psychopathology according to this RDoC initiative are, e.g., negative/positive valence systems and arousal/regulatory systems. Autonomic arousal, for instance, is positively correlated with several $\mathrm{AD}$, but also with symptoms of depression [10].

In the pathogenesis of anxiety disorders and threatresponse regulation, fear conditioning is a central learning mechanism. Differential fear conditioning (FC) refers to learning that a conditioned stimulus $(\mathrm{CS}+)$ predicts an aversive event [the unconditioned stimulus (UCS)], while another stimulus (CS -) is never followed by the UCS and predicts safety. In fear generalization, the conditioned fear responses extend to stimuli (generalization stimuli, GSs) more or less similar to the $\mathrm{CS}+$, but never associated with the UCS. The generalization gradient usually diminishes as a function of reduced similarity between GSs and CS + [11].

There are multiple ways of measuring conditioned fear responses, e.g., self-report ratings and psycho-physiological responses (e.g., skin conductance response). Self-report ratings of arousal level are commonly used for measuring emotional states in humans representing the fear intention. Skin conductance response (SCR) reflects changes in sweat gland activity that alters the electrical conductivity of the skin. As such, it is thus associated with the sympathetic nervous system and covaries with the arousal level.

Disturbances in fear learning are associated with $\mathrm{AD}$, since increased fear responses in underage participants with $\mathrm{AD}$ were found both toward CS + as well as CS- [12, 13], indicating impaired safety signal learning in anxious children. Furthermore, abnormalities in fear generalization became the foci of research, since, e.g., generalized anxiety disorder [14] and panic disorder [15] in adults were found to be characterized by greater fear generalization. This endophenotypic approach offers the opportunity to study the psychopathological development of $\mathrm{AD}$ as a group on a multilevel approach from subclinical levels with a focus on abnormal threat regulation, as well as dimensional constructs of a negative/positive valence system and arousal/regulatory system. AD [16] and fear conditioning [17] have shown heritability estimates ranging between 30 and $45 \%$. However, although many candidate gene polymorphisms have been investigated in animals and in humans relating to fear learning and AD [18-20], the genetic architecture underlying human maladaptive overgeneralization has been poorly investigated. Additionally, it remains largely unknown which susceptibility genes play a role in subjective and objective arousal during fear learning and its generalization.
Most genome-wide association studies (GWAS) have been performed for specific disorders rather than clusters of disorders with continuous liability distribution, even if there are shared genetic risk factors between disorders and substantial lifetime comorbidity [21, 22]. A meta-analysis of genome-wide association studies of $\mathrm{AD}$ in nine clinical samples of European ancestry from seven large, independent studies [23] provided a novel approach to explicitly incorporate comorbidity structure directly into prediction of single nucleotide polymorphisms (SNPs) effects using case-control contrasts and factor score analysis accounting for different ADs in the meta-analysis. This genetic study of AD identified novel genetic variants significantly associated with AD. On chromosome $2 \mathrm{p} 21$, three SNPs were detected using a factor score model: rs1067327 within CAMKMT (encoding a calmodulin-lysine $N$-methyltransferase), rs 1142523 within SLC3A1 (encoding the large subunit of a heterodimeric dibasic/neutral amino acid transporter), and rs786618 within PREPL (encoding a putative prolyl endopeptidase belonging to the prolyl oligopeptidase family). One SNP on chromosome 3q12.3 (rs1709393) within LOC152225, (encoding an uncharacterized non-coding RNA locus) was identified using case-control analysis.

In the present study, we first evaluated the findings of Otowa et al. [23] in a large sample of healthy children aged 8-12 years by investigating associations of these SNPs with anxious personality traits in a dimensional approach. In a second step, we analyzed the association of subjective and psychophysiological arousal with the genetic variants found by the meta-analysis of Otowa and colleagues, using a discriminative fear conditioning and generalization paradigm. We expected to find an association of these risk variants with anxiety-relevant traits as well as fear generalization gradients and arousal level. The sample has been recruited with the comprehensive research center "SFB/TRR58-Fear, Anxiety and Anxiety Disorders" funded by the German Research Foundation and, to our knowledge, represents the by far largest sample of children with both data on genetics and fear conditioning/generalization.

\section{Methods}

\section{Sample}

All participants were recruited from primary/secondary schools in the greater region of Wuerzburg, Germany. Inclusion criteria were Caucasian descent (self-report up to third generation), right-handedness (Edinburgh Handedness Inventory; [24]), and fluency in German. Exclusion criteria were manifest or lifetime DSM-IV axis I disorder, severe medical conditions, intake of psychoactive medication, and an IQ $<85$ as ascertained by the German version of the 
Culture Fair Intelligence Test 2 [25]. Absence of DSM-IV axis I disorder was ascertained using the German versions of the Diagnostic Interview for Mental Disorders for Children and Adolescents (Kinder-DIPS; [26]). A total of 475 children between 8 and 12 years of age participated in the study. However, 30 children did not complete the experiment ( 7 children canceled the experiment prior to acquisition, 6 canceled at acquisition, and additionally 17 children canceled at generalization). Additionally, 42 children were excluded from analysis due to failure of genetic data and/or problems with physiological recordings. Additionally, 56 children were excluded due to genetic relationships with other participants. Thus, a total of 347 children (174 females; mean age: 9.7; SD 1.3) were included in the study. All children gave $2 \times 10 \mathrm{ml}$ venous EDTA blood for genetic analyses. Additionally, all participants had to answer to fear-relevant questionnaires (see below): The German version of the Fear Survey Schedule for Children-Revised (PHOKI; [27]), the German version of the Childhood Anxiety Sensitivity Index [(KASI; ([Schneider and Silverman, 2009. Kinder-Angstsensitivitätsindex (KASI). Unpublished Manuskript]), the German version of the Trait scale of the State-Trait Anxiety Inventory for Children (STAIK-T; [Unnewehr, Joormann, Schneider, Margraf, 1992. Deutsche Übersetzung des State-Trait Anxiety Inventory for Children. Unpublished Manuskript]) as well as the German version of the Social Phobia and Anxiety Inventory for Children (SPAIK; [28]). All participants underwent a discriminant fear conditioning and generalization task. To reach a statistical power of $95 \%$, for the fear conditioning and generalization task (effect size: $f=0.025$, $\alpha$ err prob $=0.05$ ) a minimal sample size of 24 volunteers and for the psychometric traits (effect size: $d=0.85, \alpha$ err prob $=0.05$ ) a minimum of 31 participants per group are needed ( $G *$ Power v3.1.9.2). The study was approved by the ethical committee of the Medical Faculty of the Julius-Maximilian-University of Würzburg (vote $7 / 08 ; 106 / 10$ ) and complied with the latest version of the Declaration of Helsinki. All participants as well as their parents gave written informed consent and each family was paid $€ 50$ compensation for their participation.

\section{Genotyping}

For genotyping, four significant SNPs, identified in the study of Otowa et al. [23] (rs1067327, rs786618, rs1142523 and rs1709393-for localization of SNPs, see Suppl Fig. 1), were chosen for analysis. Genotyping data of rs786618, rs1142523 and rs1709393 were generated using MassARRAY system (Agena Bioscience, San Diego; for Primer sequences, see Suppl Table 1) with the iPlex ${ }^{\circledR}$ chemistry following the standard operation procedure and for rs 1067327 by restriction analysis of PCR products. PCR fragments were amplified using the primers forward 5'-GCAGGG
TAAATTCTTCATTGGT-3' and reverse 5'-CAGAAAGAG CAATCTCCACAAG- $3^{\prime}$ in a $25 \mu$ reaction mix containing $50 \mathrm{ng}$ genomic DNA, $2.5 \mu \mathrm{l}$ Gold Star buffer, $10 \mu \mathrm{M}$ of each primer, $2.5 \mathrm{mM}$ of each nucleotide, $25 \mathrm{mM} \mathrm{MgCl}_{2}$ and $0.5 \mathrm{U}$ of Taq polymerase. Cycling conditions were $2 \mathrm{~min}$ at $94{ }^{\circ} \mathrm{C}$ initial denaturation, and $30 \mathrm{~s}$ at $94{ }^{\circ} \mathrm{C}, 30 \mathrm{~s}$ at $59^{\circ} \mathrm{C}, 30 \mathrm{~s}$ at $72{ }^{\circ} \mathrm{C}$ for 35 cycles and a final extension step at $72{ }^{\circ} \mathrm{C}$ for 7 min. PCR products (size $591 \mathrm{bp}$ ) were digested with $\operatorname{Csp} \mathrm{I}$ and fragments were visualized on a $2 \%$ agarose gel. All four SNPs were polymorphic (minor allele frequency $>0.2 \%$ ), reached a minimal genotyping call rate of $>96 \%$ and did not differ from Hardy-Weinberg equilibrium $(P>0.3)$. Genotypes were determined and analyzed by investigators blinded to phenotypes of participants (sample sizes and allele frequencies are shown in Suppl Table 2).

\section{Fear-relevant psychometric traits (self-report measures)}

The PHOKI consists of 98 items on a three-point Likert scale: (0) "never", (1) "sometimes" and (2) "often". The PHOKI has seven subscales: Fear of Threats and Death (GT), Separation Anxiety (TA), Social Anxiety (SA), Fear of Weirdness (BU), Animal Phobias (TP), Fear of Medical Invasions (ME), and School- and Performance Anxiety (SL). The KASI is a self-report scale to measure the level of anxiety, fearfulness and anxiety disorders on 17 items, describing potential reactions to physical symptoms and anxiety on a three-point Likert scale (1) "never", (2) "sometimes" and (3) "often", resulting in a sum score between 17 and 51. The STAIK is a self-report scale to determine the level of trait anxiety on 20 statements on a three-point Likert scale (1) "almost never", (2) "sometimes" and (3) "often", resulting in a sum score between 20 and 60. The SPAIK is a self-report scale to determine the level of social anxiety. The SPAIK consists of 26 items on a three-point Likert scale (0) "never or almost never", (1) "sometimes" and (2) "most of the time or ever", resulting in a sum score between 0 and 52 (descriptive characteristics of fear-relevant psychometric traits of the total sample are given in Suppl Table 3).

\section{Fear conditioning and generalization task}

We used the "screaming lady paradigm" based on Lau et al. [29] and adapted by Schiele, Reinhard et al. [30]. Some studies indicate that the "screaming lady paradigm" represents a more powerful unconditioned stimulus (UCS) than other UCSs employed in research with children and adolescents [30-32].

Pictures of two actresses with neutral facial expression (NimStim Face Stimulus Set; [33]) served as either the $\mathrm{CS}+$ or $\mathrm{CS}-$, with one of the two faces being randomly selected as the CS + for each participant. The UCS was 
a 95-dB female scream (International Affective Digital Sounds system), presented simultaneously with a fearful facial expression of the same actress assigned as the CS + . Four generalization stimuli depicting gradual morphs from CS + to CS - in 20\%-steps (GS1-4) were created using the graphics software Sqirlz Morph Version 2.1 (Xiberpix, Solihull, UK). Stimulus presentation was controlled using Presentation software version 17.2 (Neurobehavioral Systems, Inc., Albany, CA, USA). CSs and GSs were presented for $6 \mathrm{~s}$ each. The UCS was presented immediately following $\mathrm{CS}+$ offset for $1.5 \mathrm{~s}$. Inter-trial intervals varied from 9 to $12 \mathrm{~s}$, during which a white fixation cross was displayed centrally on the screen. Stimulus order was pseudo-randomized so that the same stimulus could not appear more than twice in a row.

The experiment was divided into three consecutive phases: pre-acquisition, acquisition, and generalization. Pre-acquisition consisted of four $\mathrm{CS}+$ and four $\mathrm{CS}-$; no UCS was presented. During acquisition, $12 \mathrm{CS}+$ and 12 $\mathrm{CS}$ - were presented. The CS + was paired with the UCS on ten trials. The generalization phase consisted of $12 \mathrm{CS}+$, $12 \mathrm{CS}-$, and 12 of each of the four GSs. Half the CS + trials were followed by the UCS to prevent premature extinction. CS- and all GSs were never paired with the UCS. Participants were not informed of the CS-UCS contingencies. Acquisition and generalization trials were separated into two phases, each containing half the trials per phase, i.e., six presentations per stimulus category. Participants were instructed to passively view pictures of two female faces, and that an unpleasant sound would be heard occasionally. They were told that it would be possible to become startled and/or frightened and that participation could be discontinued at any time.

Following (pre)-acquisition and generalization, participants rated each stimulus on arousal and UCS expectancy. Arousals were indicated on nine-point Likert scales, ranging from "very calm" (1) to "very arousing" (9). UCS expectancy was recorded in percent on a scale from 1 to 100 in $10 \%$ increments as the probability of an aversive noise following each stimulus. Participants were considered aware of the CS-UCS relationship, if UCS expectancy ratings were higher for the $\mathrm{CS}+$ than the $\mathrm{CS}-$. Contingency awareness after acquisition and generalization was determined using the ratings after the second acquisition and generalization phase, respectively.

\section{Physiological recordings and data reduction}

Throughout the experiments, skin conductance responses (SCRs) were recorded continuously using Brainproducts V-Amp-16 and Vision Recorder software (Brainproducts, Gilching, Germany) at a sampling rate of $1000 \mathrm{~Hz}$ and analyzed off-line using Vision Analyzer 2 software
(Brainproducts, Gilching, Germany). SCR was recorded from the thenar and hypothenar eminences of the left hand using two $\mathrm{Ag} / \mathrm{AgCl}$ electrodes. The amplifier delivered a constant current of $0.5 \mathrm{~V}$. The SCR signal was filtered offline with a high cutoff filter of $1 \mathrm{~Hz}$ and a notch filter of $50 \mathrm{~Hz}$. SCR was defined as the base-to-peak difference (in $\mu \mathrm{S})$ between response onset $(900-4000 \mathrm{~ms}$ after stimulus onset) and peak (2000-6000 ms after stimulus onset). A minimum response criterion of $0.02 \mu \mathrm{S}$ was applied, with lower responses scored as 0 . SCR data were normalized following an approach described by Dunsmoor et al. [34], i.e., by computing generalization gradients for each phase and block as a function of the response to one stimulus type relative to the sum of responses to all stimuli.

\section{Statistical analyses}

All statistical tests were carried out using SPSS version 24 (SPSS Inc., Chicago, Illinois, USA).

\section{Fear-relevant psychometric traits}

Descriptive characteristics of genetic variants relative to fear-relevant psychometric traits (PHOKI, KASI, STAIK, and SPAIK) are presented in Suppl Table 4. $T$ tests were used to measure differences between the genotypes according to fear-relevant psychometric traits [PHOKI (including seven subscales and a total sum score resulting in 8 PHOKI scales), KASI, STAIK, and SPAIK]. Bonferroni correction was used to correct for multiple testing (significance threshold: $p<0.0042$, since 12 tests were performed).

\section{Fear conditioning and generalization task}

Ratings and SCR were analyzed using repeated-measures ANOVAs with the between-subject factor genotype (3 groups: rs1067327: CC vs. CG vs. GG; rs786618: CC vs. CT vs. TT; rs1142523: TT vs. CT vs. CC; rs1709393: CC vs. CT vs. TT) and the within-subject factor stimulus type (CS +/CS - at acquisition, CS +/CS -/GS1-4 at generalization). Additionally, the within-subject factor phase (phase 1 vs. phase 2) was included to detect possible reaction changes between the first and second phase. Due to significant differences in contingency awareness at generalization for rs1709393 ( $p=0.007)$, the between-subject factor awareness (unaware, aware) was included for analyses relating to rs1709393 at generalization, since awareness of the CS-UCS relationship may influence the conditioned responses [35]. Furthermore, because there were significant sex differences for rs1067327 $(p=0.009)$, rs786618 $(p=0.013)$, and rs1142523 $(p=0.022)$, sex was set as covariate of no interest for analyses concerning these SNPs. ANOVAs were followed by post hoc $t$ tests for significant interactions. Alpha 
was set at 0.05 and Bonferroni correction was applied. Greenhouse-Geisser corrections for non-sphericity were performed where indicated, though uncorrected degrees of freedom are reported for the sake of better readability. Corrected $p$ values and partial $\eta^{2}$ for relevant significant results are reported.

\section{Results}

\section{Fear-relevant psychometric traits}

Table 1 shows relevant $p$ values of the relations of genotypes and fear-relevant psychometric traits (PHOKI-TP, KASI, STAIKC, and SPAIK; for the sake of clearer arrangement, we focused on PHOKI subscale TP as the most relevant subscale here; results of the other subscales as well as PHOKI total score are presented in Suppl Table 5). For CAMKMT rs1067327 and PREPL rs786618, CC genotype carriers compared to GG as well as CT and TT genotype carriers, respectively, were significantly associated with lower scores in the fear-relevant psychometric trait PHOKI (TP: $t(38)=-3.21, p_{\text {rs } 1067327}=0.003$; TP: $t(44)=-3.0$, $p_{\mathrm{rs} 786618}=0.004$; at a nominal level in SL: $p_{\mathrm{rs} 786618}=0.017$ ). No significant associations for STAIKC and/or SPAIK were found (all $p \mathrm{~s} \geq 0.167$ ). For $S L C 3 A 1 \mathrm{rs} 1142523$, at a nominal level CC genotype carriers compared to TT genotype carriers were significantly associated with higher PHOKI scores (TP: $t(48)=2.69, p=0.010)$, conveying genetic risk. Relations with other fear-relevant psychometric traits were not significant (all $p \mathrm{~s} \geq 0.064$ ). LOC152225 rs 1709393 reached no significance at all (all $p \mathrm{~s} \geq 0.046$ ).

\section{Fear conditioning and generalization task}

We identified differences in fear learning and generalization according to subjective and psychophysiological arousal in relation to the underlying genetic background, comparing three genotype groups per SNP, respectively, during the acquisition and generalization phases.

\section{Results during acquisition}

Arousal ratings for all relevant SNPs confirmed that the expected conditioning effect as arousal for the CS + was higher than for the CS - (all $p s \leq 0.100$ ). For $P R E P L$ rs786618, the main effect of genotype reached significance $(p=0.027)$, indicating lower arousal in minor allele carriers and higher arousal in major allele carriers, respectively. SCR for LOC152225 rs1709393 also confirmed the expected conditioning effect as the SCRs to CS + were higher than
Table $1 P$ values of relations of fear-relevant psychometric traits and genotypes

\begin{tabular}{|c|c|c|c|c|c|c|c|c|}
\hline & PHOKI (TP) $M(\mathrm{SD})$ & $p$ value & KASI $M(\mathrm{SD})$ & $p$ value & STAIK & $p$ value & SPAIK & $p$ value \\
\hline \multicolumn{9}{|c|}{ CAMKMT rs 1067327} \\
\hline $\mathrm{CC}$ & $2.44(2.28)$ & 0.003 & $24.67(3.97)$ & 0.161 & $28.07(6.25)$ & 0.281 & $8.85(6.87)$ & 0.166 \\
\hline $\mathrm{CG}$ & $3.99(3.47)$ & & $25.84(5.83)$ & & $29.43(6.19)$ & & $11.23(8.35)$ & \\
\hline GG & $4.00(3.69)$ & & $25.87(5.64)$ & & $29.46(6.50)$ & & $10.86(8.79)$ & \\
\hline \multicolumn{9}{|c|}{$P R E P L$ rs 786618} \\
\hline $\mathrm{CC}$ & $2.57(2.39)$ & 0.004 & $24.50(4.04)$ & 0.076 & $27.90(6.01)$ & 0.122 & $9.70(7.34)$ & 0.356 \\
\hline $\mathrm{CT}$ & $4.17(3.80)$ & & $25.97(5.93)$ & & $29.25(6.20)$ & & $10.84(8.34)$ & \\
\hline TT & $3.88(3.58)$ & & $25.97(5.60)$ & & $29.90(6.54)$ & & $11.32(8.99)$ & \\
\hline \multicolumn{9}{|c|}{ SLC3A1 rs 1142523} \\
\hline TT & $2.67(2.50)$ & 0.010 & $25.37(6.00)$ & 0.545 & $28.26(6.39)$ & 0.257 & $10.70(7.73)$ & 0.650 \\
\hline CT & $3.90(3.50)$ & & $25.68(5.63)$ & & $28.98(6.26)$ & & $10.71(8.44)$ & \\
\hline $\mathrm{CC}$ & $4.18(3.88)$ & & $26.07(5.69)$ & & $29.77(6.43)$ & & $11.15(8.79)$ & \\
\hline \multicolumn{9}{|c|}{ LOC152225 rs1709393 } \\
\hline $\mathrm{CC}$ & $3.17(3.10)$ & 0.072 & $24.88(4.29)$ & 0.142 & $28.21(5.69)$ & 0.148 & $9.11(7.49)$ & 0.123 \\
\hline CT & $4.20(3.75)$ & & $25.90(5.78)$ & & $29.61(6.82)$ & & $11.13(8.69)$ & \\
\hline TT & $3.60(3.21)$ & & $25.98(5.85)$ & & $29.55(5.85)$ & & $11.14(8.30)$ & \\
\hline
\end{tabular}

An additive model was used to pertain the $p$ values; the above-named $p$ values refer to differences between phenotypes that are furthest apart from each other

$M$ mean, $S D$ standard deviation, $P H O K I$ Fear Survey Schedule for Children-Revised (German version), subscale TP Animal Phobia, KASI Childhood Anxiety Sensitivity Index (German version), STAIK Trait Scale of the State-Trait Anxiety Inventory for Children (German version), SPAIK Social Phobia and Anxiety Inventory for Children (German version)

$* p<.0042 ; p$-values that survived Bonferroni correction for multiple testing are highlighted in bold 
SCRs to CS - $(p<0.001)$. Additionally, phase reached a significant main effect $(p<0.001)$ with lower SCR in phase 2. Following up a significant Stimulus Type $\mathrm{x}$ Phase interaction for LOC152225 rs $1709393(F(1,253)=6.08, p=0.014$, $\eta^{2}=0.02$ ), results showed higher differences between the stimuli in phase 2 indicating better cue discrimination in phase 2 .

\section{Results during generalization}

Significant main effects of stimulus type for all relevant SNPs indicated upward generalization gradients from $\mathrm{CS}+$ to $\mathrm{CS}-$ according to arousal ratings (all $p s \leq 0.032$ ) as well as SCR (all $p s<0.001$ ). For CAMKMT rs 1067327 and SLC3A1 rs1142523 (and marginally for PREPL rs786618: $p=0.096$ ), significant main effects of genotypes were found in arousal ratings (CAMKMT rs1067327: $F(2,336)=3.08$, $p=0.047, \eta^{2}=0.02$; SLC3A1 rs1142523: $F(2,337)=3.34$, $\left.p=0.036, \eta^{2}=0.02\right)$. For CAMKMT rs 1067327 , higher arousal in GG genotype carriers (Fig. 1a) (and in TT genotype carriers for PREPL rs786618, respectively; Fig. 1b) was found, and higher arousal in CC genotype carriers was found for SLC3A1 rs1142523 (Fig. 1c). Additionally, for PREPL rs786618, a significant Genotype $\mathrm{x}$ Phase interaction effect was found for $\operatorname{SCR}(F(2,246)=3.83, p=0.023$, $\eta^{2}=0.02$ ). Post hoc tests, however, reached no significance. No significant main effect was found for genotypes related to LOC152225 rs 1709393 (Fig. 1).

For arousal ratings, contingency awareness played a significant role within the analyses with a Phase $\times$ Genotype $\times$ Awareness interaction $(F(2,328)=3.25, p=0.040$, $\eta^{2}=0.02$ ), a significant main effect of awareness, and a significant Stimulus Type $\times$ Awareness interaction resulting in a three-way interaction of Stimulus Type $\times$ Phase $\times$ Awareness for LOC152225 rs1709393 $(F(5,1516)=8.70$, $\left.p<0.001, \eta^{2}=0.03\right)$. Post hoc tests indicated that aware participants were better at discriminating stimuli compared to unaware participants, and this was true for phase 1 and phase 2, but with better stimuli discrimination of aware participants in phase 2, and with TT genotype carriers being more often aware than $\mathrm{CC}$ genotype carriers. Furthermore, awareness played also a role in Arousal ratings as indicated by a significant main effect of awareness for LOC 152225 rs1709393 $\left(F(1,328)=8.44, p=0.004, \eta^{2}=0.02\right)$ with higher SCR in aware participants.

\section{Discussion}

The present study investigated the impact of four genetic risk variants, recently identified as associated with pathological anxiety in adults in a meta-analysis of genome-wide association studies of anxiety disorders (AD) [23], on fearand anxiety-relevant personality traits in a large sample of typically developing children aged 8 to 12 years. In this study, we aimed to extend the findings of Otowa et al. [23] by applying a dimensional approach and including physiological conditioning/generalization data. The chosen age range marks a developmental period, in which a wide range of AD manifest for the first time. Assuming that common variants play a crucial role in AD susceptibility, we additionally analyzed those risk variants with genome-wide significance [23] in healthy children with respect to generalization gradients according to subjective and objective measures of arousal, since overgeneralization and heightened arousal are assumed risk factors in the pathogenesis of $\mathrm{AD}[12,14$, $15,36]$.

First, we evaluated the findings by Otowa et al. [23] in a sample of healthy $8-12$ years old children in a dimensional approach, indicating correlations of fear- and anxiety-relevant personality traits and heightened anxiety sensitivity with the analyzed risk genotypes. In more detail, analyses of the used fear- and anxiety-relevant dimensional personality traits showed that relating to CAMKMT rs1067327, PREPL rs786618, and at a nominal level $S L C 3 A 1$ rs1142523, the minor allele was associated with lower scores in PHOKI (basically TP) and, thus, the minor allele functioned more in a protective manner. The other dimensional questionnaires yielded similar effects, but reached no significance, presumably due to lack of power and/or little variance. We could only establish the link in chromosome 2, but reached no significance for chromosome 3 , again possibly due to small sample sizes and due to the little diverse dimensional phenotypes. On the other hand, the SNP in chromosome 3 may not be associated with alterations in arousal, but with other $\mathrm{RDoC}$, such as for example valence. Thus, further exploration is necessary. The reason for the sole genetic association with the PHOKI scores might possibly be due to the age of the participants. Psychopathological differentiation between categorical disorders in children is often hampered by the child's difficulty differentiating distinct constructs and expressing themselves. Thus, while KASI and STAIK measure an anxiety-prone personality and SPAIK scores assess social anxious traits, the PHOKI relates to specific and clear-cut fears that may be readily distinguishable for children.

Further, we found that the analyzed risk genotypes on chromosome 2 are associated with altered arousal to threat/safety stimuli during fear learning, but not to enhanced generalization per se. In detail, while for the overall sample conditioning was successful and the expected upward generalization gradients from CS + to $\mathrm{CS}-$ could be identified, there were main effects of genotypes for CAMKMT rs1067327 and SLC3A1 rs1142523, 
(A)

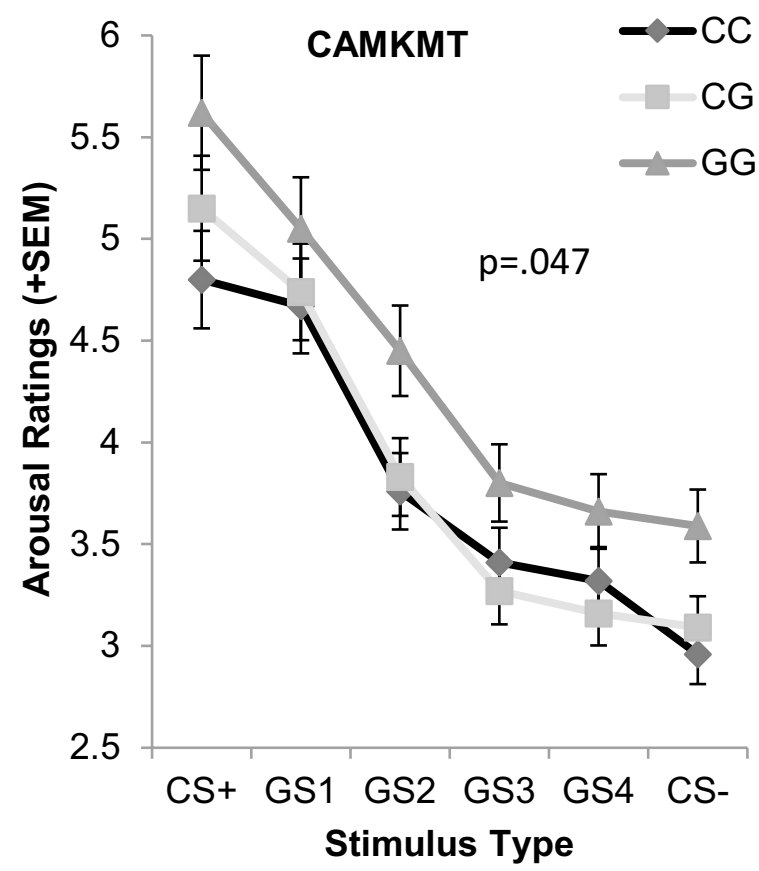

(B)

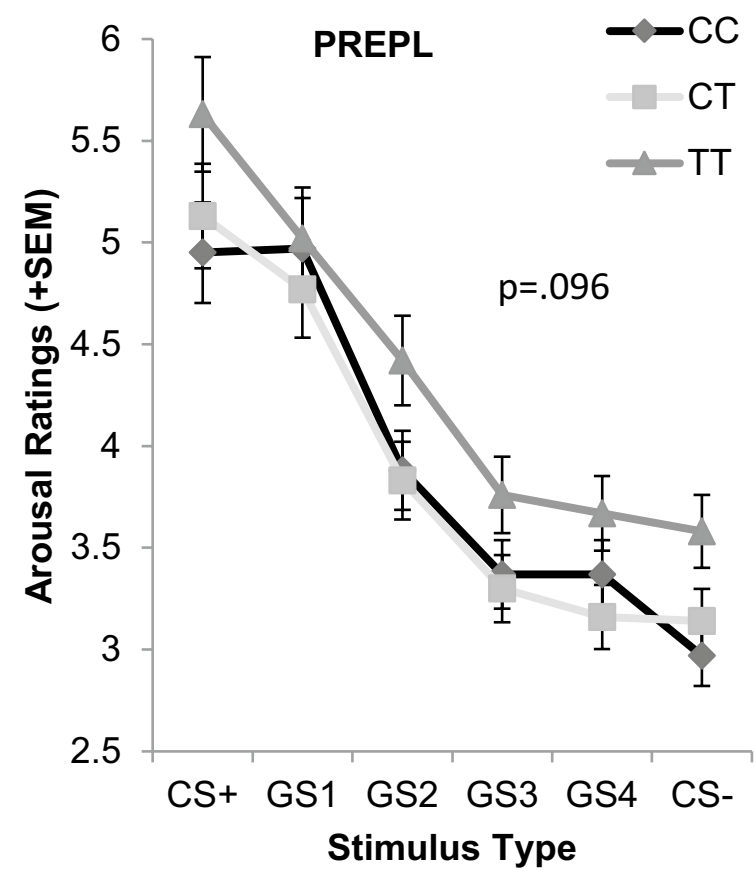

(C)

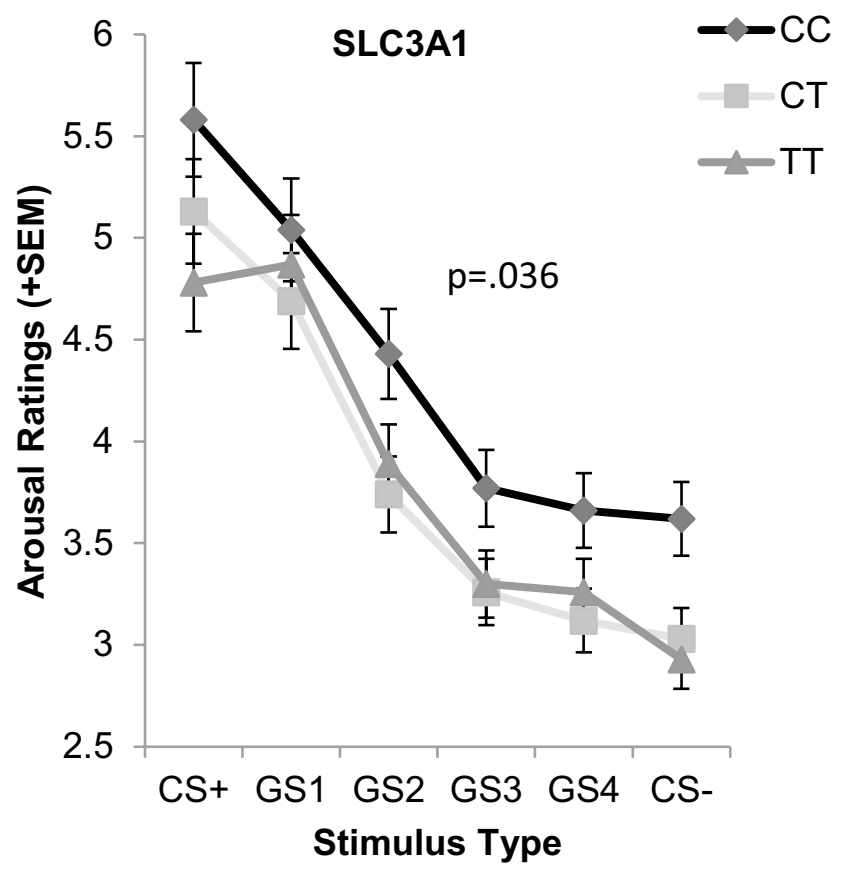

(D)

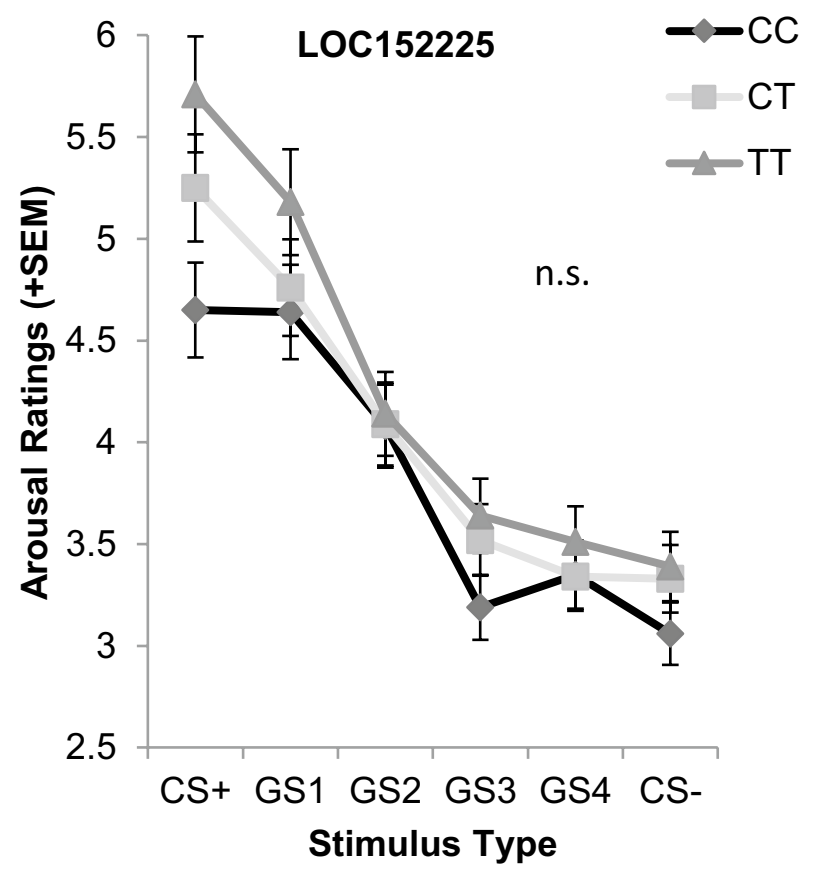

Fig. 1. Fear generalization gradients according to arousal ratings for a CAMKMT rs1067327 genotypes, b PREPL rs786618 genotypes, c SLC3A1 rs1142523 genotypes, and d LOC152225 rs1709393 genotypes 
but no Genotype $\times$ Stimulus Type interaction effects. The homozygote genotypes with major allele frequency (GG in CAMKMT rs 1067327, TT in PREPL rs786618, CC in SLC3A1 rs1142523) were consistently associated with higher arousal ratings. However, results according to SCR yielded no significant effects, possibly due to larger physiological variance in children and the high error rate for physiological measures of arousal, although similar trends were found. Our results are in line with previous studies showing that anxious participants rated emotional images as more arousing and reported more fear than healthy participants, and that there were inconsistencies between self-reports and physiological states $(12,37,38]$. However, our results must be interpreted carefully, since even though the here presented sample size represents the largest child sample to date with combined genetic and fear conditioning/generalization data, it is still comparatively small for investigating genetic effects. Thus, results must be analyzed and reviewed in larger samples, integrating healthy as well as pathologically anxious participants.

Moreover, consistent with previous results of our research group [30], participants, who were aware of the contingency, were better at stimuli discrimination and this was especially true for phase 2 . Additionally, aware participants showed generally higher SCR than unaware participants and TT genotype carriers were more often aware than CC genotype carriers according to LOC 152225 rs1709393. This finding could be explained by attentional processes, meaning that TT genotype carriers showed more attention toward threat. This would match a study showing that clinically anxious participants consistently shifted attention toward threat, resulting in reduced detection latencies for probes appearing in the vicinity of such stimuli, supporting the existence of anxiety-related encoding bias that may contribute to the maintenance of such mood disorders [37]. However, this finding must be verified in larger samples with and without $A D$.

The results expand results of the named meta-analysis of genome-wide association studies of $\mathrm{AD}$, but require replication and further exploration in other samples. Importantly, the effect found in our data regarding rs1067327 was contrarious to what is specified in the meta-analysis of Otowa et al. [23]. Whereas in our sample, anxiety traits and increased arousal were positively associated with the major $\mathrm{G}$ allele, the results of Otowa et al. [23] indicated a positive association between the $\mathrm{C}$ allele and the corresponding anxiety phenotype. This could be due, for instance, to different samples. Whereas in the named meta-analysis adults were included, we exclusively investigated children. Additionally, children of our study were healthy, whereas participants with different manifest anxiety disorders were included in the named meta-analyses. Furthermore, a reduction of variation could have occurred due to more anxious children cancelling the experiment and thus having reduced our liability scale on the upper anxiety end. Indeed, the 30 children that did cancel were significantly more anxious relating to STAIK scores than the other participants, but relating to other self-report measures and also to genotype there were no statistical differences between groups. Unfortunately, we were unable to determine the directions of the associations between rs786618 as well as rs1142523 and the corresponding anxiety phenotype as described in Otowa et al. [23].

How could the three SNPs from the chromosome $2 \mathrm{p} 21$ region be mechanistically linked to the development of increased anxiety sensitivity and higher arousal states during fear learning? All three SNPs are located in a very generich region spanning only approximately $83 \mathrm{~kb}$ (see Suppl Fig. 1) and are therefore not inherited independently, thus not allowing to attribute the association to a specific gene. The rs1142523 and rs1067327 SNPs are located in intronic regions in their respective genes (SLC3A1 and CAMKMT), whereas rs 786618 is located in the $5^{\prime} \mathrm{UTR}$ of PREPL. It is not known if any of these SNPs influence the splicing pattern, expression levels, or transcript stability of their respective genes. Interestingly, a yet uncharacterized microRNA gene is located intronic in CAMKMT as well. According to databases, all these transcripts can be detected in brain tissue. In humans a deletion of the $2 \mathrm{p} 21$ region including four genes (PP2C $\beta, S L C 3 A 1, P R E P L$ and CAMKMT) was described as a syndrome (MIM \#606,407) associated with cystinuria, intellectual disability, mitochondrial disease, hypotonia and facial dysmorphism [38]. Mice deficient for CAMKMT expression show developmental delay, mitochondrial defects, and brain alterations [39]. Motor learning and complex coordination and learning of aversive stimuli are also impaired, pointing to the possibility that CAMKMT could be a reasonable candidate for further experiments. Knockout mice for PREPL suffer neonatal hypotonia and diminished growth [40] making this gene a less likely candidate, similarly to $S L C 3 A 1$ null mice displaying cystinuria [41]. Further experiments are needed to investigate how these human SNPs might be associated with anxiety and arousal and what biological mechanisms are associated with this phenotype.

Taken together, our results expand findings by Otowa et al. [23] evaluating the findings on adult patient samples in healthy children aged 8-12 years and showing that the anxiety disorder risk SNPs are associated both with (1) dimensional anxiety traits and (2) arousal. As this is the first study evaluating the findings by Otowa et al. [23] in a dimensional approach in a children sample, our results must be replicated in larger samples and with other robust approaches. These should also include additional genome-wide significant SNPs generated in a very recent study investigating more than 150.000 individuals in the UK [42], or by including 
SNPs identified in a recent twin study in generalized anxiety disorder [43]. Heightened anxiety traits and arousal could serve as trans-diagnostic frameworks in terms of the RDoC approach and accelerate the definition of the genetic underpinnings of anxiety disorders.

Acknowledgements The authors would like to thank the excellent technical assistance of Carola Gagel and Zuzana Fouskova.

Funding This study was supported by a grant from the Deutsche Forschungsgemeinschaft (DFG; SFB-TRR58, projects B01 to PP, C02 to $\mathrm{KD}$ and $\mathrm{JD}$, and $\mathrm{Z} 02$ to JD, $\mathrm{AR}, \mathrm{KD}, \mathrm{PP}$, and MR).

\section{Compliance with ethical standards}

Conflict of interest On behalf of all authors, the corresponding author states that there is no conflict of interest.

Open Access This article is licensed under a Creative Commons Attribution 4.0 International License, which permits use, sharing, adaptation, distribution and reproduction in any medium or format, as long as you give appropriate credit to the original author(s) and the source, provide a link to the Creative Commons licence, and indicate if changes were made. The images or other third party material in this article are included in the article's Creative Commons licence, unless indicated otherwise in a credit line to the material. If material is not included in the article's Creative Commons licence and your intended use is not permitted by statutory regulation or exceeds the permitted use, you will need to obtain permission directly from the copyright holder. To view a copy of this licence, visit http://creativecommons.org/licenses/by/4.0/.

\section{References}

1. Jacobi F, Höfler M, Siegert J, Mack S, Gerschler A, Scholl L et al (2014) Twelve-month prevalence, comorbidity and correlates of mental disorders in Germany: the Mental Health Module of the German Health Interview and Examination Survey for Adults (DEGS1-MH). Int J Methods Psychiatr Res 23(3):304-319

2. Kessler RC, Amminger GP, Aguilar-Gaxiola S, Alonso J, Lee S, Üstün TB (2007) Age of onset of mental disorders: a review of recent literature. Curr Opin Psychiatry 20(4):359-364

3. Beesdo-Baum K, Knappe S, Asselmann E, Zimmermann P, Brückl T, Höfler M et al (2015) The 'Early developmental stages of psychopathology (EDSP) study': a 20-year review of methods and findings. Soc Psychiatry Psychiatr Epidemiol 50(6):851-866

4. Regier DA, Rae DS, Narrow WE, Kaelber CT, Schatzberg AF (1998) Prevalence of anxiety disorders and their comorbidity with mood and addictive disorders. Br J Psychiatry 173(34):24-28

5. Pine DS (1997) Childhood anxiety disorders. Curr Opin Pediatr 9:329-338

6. Craske MG, Rauch SL, Ursano R, Prenoveau J, Pine DS, Zinbarg RE (2009) What is an anxiety disorder? Depress. Anxiety 26(12):1066-1085

7. Moras K, Barlow DH 1992 Dimensionale Diagnose und das Problem von Angst und Depression. In: Fiegenbaum W, Margraf J, Florin I, Ehlers A (eds) Zukunftsperspektiven der Klinischen Psychologie. Springer, Berlin, Heidelberg.

8. Lebeau RT, Glenn DE, Hanover LN, -Baum K, Wittchen HU, Craske MG (2012) A dimensional approach to measuring anxiety for DSM-5. Int J Methods Psychiatr Res 21(4):258-272
9. Sanislow CA, Pine DS, Quinn KJ, Kozak MJ, Garvey MA, Heinssen RK et al (2010) Developing constructs for psychopathology research: research domain criteria. J Abnorm Psychol 119(4):631

10. Brown TA, Chorpita BF, Barlow DH (1998) Structural relationships among dimensions of the DSM-IV anxiety and mood disorders and dimensions of negative affect, positive affect, and autonomic arousal. J Abnorm Psychol 107(2):179

11. Ghirlanda S, Enquist M (2003) A century of generalization. Anim Behav 66:15-36

12. Britton JC, Grillon C, Lissek S, Norcross MA, Szuhany KL, Chen $G$ et al (2013) Response to learned threat: an fMRI study in adolescent and adult anxiety. Am J Psychiatry 170(10):1195-1204

13. Waters AM, Henry J, Neumann DL (2009) Aversive Pavlovian conditioning in childhood anxiety disorders: impaired response inhibition and resistance to extinction. J Abnorm Psychol 118(2):311-321

14. Lissek S, Kaczkurkin AN, Rabin S, Geraci M, Pine DS, Grillon C (2014) Generalized anxiety disorder is associated with overgeneralization of classically conditioned fear. Biol Psychiat 75:909-915

15. Lissek S, Rabin S, Heller RE, Lukenbaugh D, Geraci M, Pine DS et al (2010) Overgeneralization of conditioned fear as a pathogenic marker of panic disorder. Am J Psychiatry 167:47-55

16. Hettema JM, Neale MC, Kendler KS (2001) A review and metaanalysis of the genetic epidemiology of anxiety disorders. Am J Psychiatry 158:1568-1578

17. Hettema JM, Annas P, Neale MC, Kendler KS, Fredrikson M (2003) A twin study of the genetics of fear conditioning. Arch Gen Psychiatry 60(7):702-708

18. Chen ZY, Jing D, Bath KG, Ieraci A, Khan T, Siao CJ et al (2006) Genetic variant BDNF (Val66Met) polymorphism alters anxietyrelated behavior. Science 314(5796):140-143

19. Lonsdorf TB, Weike AI, Golkar A, Schalling M, Hamm AO, Öhman A (2010) Amygdala-dependent fear conditioning in humans is modulated by the BDNFval66met polymorphism. Behav Neurosci 124(1):9-15

20. Mühlberger A, Andreatta M, Ewald H, Glotzbach-Schoon E, Tröger C, Baumann C et al (2014) The BDNF Val66Met polymorphism modulates the generalization of cued fear responses to a novel context. Neuropsychopharmacology 39(5):1187

21. Hettema JM, Prescott CA, Myers JM, Neale MC, Kendler KS (2005) The structure of genetic and environmental risk factors for anxiety disorders in men and women. Arch Gen Psychiatry 62:182-189

22. Middeldorp CM, Cath DC, van Dyck R, Boomsma D (2005) The co-morbidity of anxiety and depression in the perspective of genetic epidemiology. A review of twin and family studies. Psychol Method 35:611-624

23. Otowa T, Hek K, Lee M, Byrne EM, Mirza SS, Nivard MG et al (2016) Meta-analysis of genome-wide association studies of anxiety disorders. Mol Psychiatry 21:1485

24. Oldfield RC (1971) The assessment and analysis of handedness: the Edinburgh Inventory. Neuropsychologia 9:97-113

25. Weiss RH (2006) Grundintelligenztest Skala 2-Revision-(CFT 20-R). Hogrefe, Göttingen

26. Schneider S, Unnewehr S, Margraf J 2009 Kinder-DIPS für DSMIV-TR. Diagnostisches Interview bei psychischen Störungen im Kindes- und Jugendalter. 2nd edn. Springer, Heidelberg.

27. Döpfner M, Schnabel M, Goletz H, Ollendick TH (2006) PHOKI Phobiefragebogen für Kinder und Jugendliche. Manual. Hogrefe, Göttingen

28. Melfsen S, Florin I, Warnke A (2001) Sozialphobie- und Angstinventar für Kinder (SPAIK). Hogrefe, Göttingen

29. Lau JY, Lissek S, Nelson EE, Lee Y, Roberson-Nay R, Poeth K et al (2008) Fear conditioning in adolescents with anxiety disorders: results from a novel experimental paradigm. J Am Acad Child Adolesc Psychiatry 47:94-102 
30. Schiele MA, Reinhard J, Reif A, Domschke K, Romanos M, Deckert J, Pauli P (2016) Developmental aspects of fear: comparing the acquisition and generalization of conditioned fear in children and adults. Dev Psychobiol 58(4):471-481

31. Lau JY, Britton JC, Nelson EE, Angold A, Ernst M, Goldwin M et al (2011) Distinct neural signatures of threat learning in adolescents and adults. PNAS 108(11):4500-4505

32. Schmitz A, Merikangas K, Swendsen H, Cui L, Heaton L, Grillon C (2011) Measuring anxious responses to predictable and unpredictable threat in children and adolescents. J Exp Child Psychol 110(2):159-170

33. Tottenham N, Tanaka JW, Leon AC, McCarry T, Nurse M, Hare $T$ et al (2009) The NimStim set of facial expressions: judgments from untrained research participants. Psychiatry Res 168:242-249

34. Dunsmoor JE, Prince SE, Murty VP, Kragel PA, LaBar KS (2011) Neurobehavioral mechanisms of human fear generalization. NeuroImage 55(4): 1878-1888

35. Lovibond PF, Shanks DR (2002) The role of awareness in Pavlovian conditioning: empirical evidence and theoretical implications. J Exp Psychol Anim Behav Process 28:3-26

36. Rosebrock LE, Hoxha D, Norris C, Cacioppo JT, Gollan JK (2017) Skin conductance and subjective arousal in anxiety, depression, and comorbidity: implications for affective reactivity. J Psychophysiol 31(4):145-157

37. MacLeod C, Mathews A, Tata P (1986) Attentional bias in emotional disorders. J Abnorm Psychol 95(1):15

38. Chabrol B, Martens K, Meulemans S, Cano A, Jaeken J, Matthijs $\mathrm{G}$ et al (2008) Deletion of C2orf34, PREPL and SLC3A1 causes atypical hypotonia-cystinuria syndrome. J Med Genet 45(5):314-318

39. Haziza S, Magnani R, Lan D, Keinan O, Saada A, Hershkovitz E et al (2015) Calmodulin methyltransferase is required for growth, muscle strength, somatosensory development and brain function. PLoS Genet 11(8):e1005388

40. Lone AM, Leidl M, McFedries AK, Horner JW, Creemers J, Saghatelian A (2014) Deletion of prepl causes growth impairment and hypotonia in mice. PLoS ONE ONE 9(2):e89160

41. Livrozet M, Vandermeersch S, Mesnard L, Thioulouse E, Jaubert J, Boffa JJ et al (2014) An animal model of type a cystinuria due to spontaneous mutation in 129S2/SvPasCrl mice. PLoS ONE ONE 9(7): 102700

42. Purves KL, Coleman JRI, Rayner C, Hettema JM, Deckert J, McIntosh AM et al 2017 The Common Genetic Architecture of Anxiety Disorders. bioRxiv.

43. Davies MN, Verdi S, Burri A, Trzaskowski M, Lee M, Hettema JM et al (2015) Generalised anxiety disorder-a twin study of genetic architecture, genome-wide association and differential gene expression. PLoS ONE ONE 10(8):e0134865

44. Lissek S, Grillon C (2012) Learning Models of PTSD. In: Beck JG, Sloan DM (eds) The Oxford handbook of traumatic stress disorders. Oxford University Press, New York, pp 175-190

45. Hoehn-Saric R, McLeod DR (2000) Anxiety and arousal: physiological changes and their perception. J Affect Disord 61(3):217-224 